\title{
Aplicação de processos físico-químicos como alternativa de pré e pós-tratamento de lixiviados de aterros sanitários
}

\section{Physical-chemical processes as an alternative to pre-and post-treatment of landfill leachate}

\begin{abstract}
Luciano Matos Queiroz
Doutor em Engenharia Hidráulica e Saneamento pela Escola Politécnica da Universidade de São Paulo (USP). Professor Adjunto do Departamento de Engenharia Ambiental da Escola Politécnica da Universidade Federal da Bahia (UFBA)
\end{abstract}

\author{
Mailer Sene Amaral \\ Mestre em Engenharia Hidráulica e Saneamento pela Escola Politécnica da USP
}

Dione Mari Morita

Doutora pela Escola Politécnica da USP. Livre Docente pela Escola Politécnica da USP. Professora Associada do Departamento de Engenharia Hidráulica e

Ambiental da Escola Politécnica da USP

\section{Suher Carolina Yabroudi}

Mestre em Engenharia Química pela Universidad Del Zulia - Venezuela. Doutoranda em Engenharia Hidráulica e Sanitária pela Escola Politécnica da USP

\section{Pedro Alem Sobrinho}

Doutor pela Escola Politécnica da USP. Livre Docente pela Escola Politécnica da USP. Professor Titular da Área de Saneamento da Escola Politécnica da USP

\section{Resumo}

O presente artigo objetivou avaliar a aplicação da operação de stripping como pré-tratamento de um lixiviado de aterro sanitário visando à redução da carga de amônia afluente a um sistema biológico de tratamento, bem como, a utilização do processo de coagulação-floculação-sedimentação para a remoção da matéria orgânica recalcitrante de um lixiviado de aterro pré-tratado em um sistema de lodo ativado. As taxas de remoção de nitrogênio amoniacal variaram entre 3,5 e 3,6 mg N-NH $3 \cdot L^{-1} \cdot h^{-1}$ para o sistema de stripping composto por um reator submetido à agitação mecânica e entre 3,2 e $3,3 \mathrm{mg} \mathrm{N}-\mathrm{NH}_{3} \cdot \mathrm{L}^{-1} \cdot \mathrm{h}^{-1}$ para o reator submetido à aeração com bolhas grossas. Quanto ao pós-tratamento, os resultados obtidos indicaram que são requeridas dosagens acima de $400 \mathrm{mg} \mathrm{Fe}^{+3} \cdot \mathrm{L}^{-1}$ e $400 \mathrm{mg} \mathrm{Al}{ }^{+3} \cdot \mathrm{L}^{-1}$, para a obtenção de eficiências de remoção de matéria orgânica recalcitrante superiores a $90 \%$.

Palavras-chave: aterro sanitário; lixiviado; tratamento físico-químico.

\begin{abstract}
The aim of this article was evaluate the implementation of the ammonia stripping as a pretreatment for landfill leachate and the use of the coagulationflocculation-sedimentation process for the removal of refractory organic compounds present in a landfill leachate pretreated in an activated sludge system. The rates of ammonia nitrogen removal ranged between 3.5 and $3.6 \mathrm{mg} \mathrm{NH}_{3}-\mathrm{N} \cdot \mathrm{L}^{-1} \cdot \mathrm{h}^{-1}$ in a lab scale reactor (working volume of $20 \mathrm{~L}$ ) equipped with a mechanical stirrer and 3.2 and $3.3 \mathrm{mg} \mathrm{NH}_{3}-\mathrm{N} \cdot \mathrm{L}^{-1} \cdot \mathrm{h}^{-1}$ in a lab scale aerated column (working volume of $10 \mathrm{~L}$ ). It was possible to achieve removals above $90 \%$ of refractory organic compounds (measured as apparent color) applied coagulant dosage about $400 \mathrm{mg} \mathrm{Fe}^{+3} \cdot \mathrm{L}^{-1}$ and $400 \mathrm{mg} \mathrm{Al}^{+3} \cdot \mathrm{L}^{-1}$.
\end{abstract}

Keywords: sanitary landfill; leachate; physical-chemical treatment. 


\section{Introdução}

Aterro sanitário pode ser definido como uma tecnologia utilizada para a disposição de resíduos sólidos no solo, particularmente lixo domiciliar, fundamentado em critérios de engenharia e normas operacionais específicas, permitindo um confinamento seguro em termos de controle ambiental e proteção à saúde pública (IPT/CEMPRE, 2000). Segundo a mais recente Pesquisa Nacional de Saneamento Básico (IBGE, 2008) aproximadamente 30\% dos municípios brasileiros adota o aterro sanitário como tecnologia de tratamento e disposição final dos resíduos sólidos municipais.

A decomposição microbiológica da massa de resíduos aterrada produz alguns gases, além de um líquido de cor escura e alto poder poluidor denominado lixiviado. O gás do aterro pode ser drenado através de tubulações adequadas e encaminhado para queima ou eventual aproveitamento energético. O lixiviado, porém, é um problema mais complexo (ALEM SOBRINHO e POVINELLI, 2009).

A escolha de um processo de tratamento para lixiviados de aterros sanitários, prioritariamente, deve levar em consideração as características do líquido percolado. Essas características podem variar tanto espacialmente, quanto ao longo do tempo (aterros novos e aterros maduros), devendo-se avaliar, também, aspectos legais, custos e tecnologias disponíveis.

Sob o ponto de vista técnico, os processos de tratamento podem ser físico-químicos ou biológicos, ou ainda uma combinação de ambos. Apesar de apresentar vantagens, principalmente quanto aos custos de implantação, o tratamento biológico (aeróbio, anaeróbio ou associação de ambos) ainda necessita de melhorias, principalmente no que concerne à remoção do nitrogênio amoniacal ( $\left.\mathrm{N}-\mathrm{NH}_{3}\right)$; metais pesados e compostos recalcitrantes; além da superação de algumas dificuldades operacionais (excessiva formação de espuma, tratamento e disposição do lodo e redução da toxicidade do efluente final) (AMARAL et al., 2008).

Por sua vez, os processos físico-químicos têm sido aplicados para redução de parte da carga de $\mathrm{N}_{-} \mathrm{NH}_{3}$ à montante de sistemas biológicos de tratamento ou para remoção de compostos recalcitrantes, embora poucos trabalhos refiram-se à remoção de toxicidade do efluente final. Dentre os processos existentes (stripping da amônia, coagulação-floculação, filtração, adsorção, precipitação química e combinação desses) nenhum pode ser consagrado como melhor tecnologia aplicável devido a variabilidade do lixiviado gerado em diferentes aterros. Entretanto, melhores eficiências têm sido alcançadas com a combinação de processos físico-químicos e biológicos (KURNIAWAN, LO e CHAN, 2006).

Este artigo objetivou avaliar a aplicação da operação de stripping como alternativa de pré-tratamento de um lixiviado de aterro sanitário, visando, sobretudo, à redução da carga de nitrogênio amoniacal afluente a um sistema biológico de tratamento (experimento 1); bem como, a utilização do processo de coagulação-floculação- sedimentação (experimento 2) para a remoção da matéria orgânica recalcitrante presente em um lixiviado de aterro pré-tratado em um sistema de lodo ativado.

\section{Material e métodos}

\section{Experimento 1 - Pré-tratamento através da operação de stripping da amônia}

Para a condução da pesquisa foi utilizado um lixiviado gerado no aterro localizado no Km 26 da rodovia dos Bandeirantes, no estado de São Paulo, conhecido como aterro Bandeirantes. Desde o início da sua operação, esse aterro sanitário recebeu mais de 23 milhões de toneladas de resíduos. Ao encerrar suas atividades (março de 2007), o volume de resíduos recebido era de $7.000 \mathrm{t}^{\mathrm{d}} \mathrm{d}^{-1}$. Desse montante, $60 \%$ eram resíduos orgânicos e a vazão de lixiviado gerado aproximava-se de $1.500 \mathrm{~m}^{3} \cdot \mathrm{d}^{-1}$. Para condução da presente pesquisa, o lixiviado foi coletado semanalmente, armazenado em galões de $20 \mathrm{~L}$, transportado para o laboratório de Saneamento da Escola Politécnica da Universidade de São Paulo e mantido sob refrigeração a $4^{\circ} \mathrm{C}$.

Para avaliação do processo de stripping da amônia presente no lixiviado, foram concebidas duas configurações distintas. A primeira consistia de um reator construído em chapas de acrílico (2,0 mm de espessura) com dimensões internas de $25 \times 25 \times 40 \mathrm{~cm}$ (volume útil igual a $20 \mathrm{~L})$ mantido sob agitação $\left(\mathrm{G}=340 \mathrm{~s}^{-1}\right)$ através de um agitador mecânico de palhetas simples (Fuzzylogie, modelo CAT R100C). No segundo sistema de tratamento, a operação de stripping era conduzida em um reator cilíndrico com diâmetro interno igual a 14,0 cm, também construído em chapas de acrílico (2,0 mm de espessura) cujo conteúdo era mantido sob agitação através de um sistema de aeração composto por um pequeno compressor e um dispersor de ar (bolhas grossas) imerso na coluna líquida. A altura da coluna de lixiviado mantida sob aeração (altura para a transferência de oxigênio) era igual a 65,0 cm. A vazão de ar $\left(30\right.$ L.h $\left.^{-1}\right)$ era controlada por um rotâmetro (marca Conault com vazão de ar máxima de 35 L.h-1 ${ }^{-1}$.

A utilização da operação de stripping para redução da carga de amônia de lixiviados de aterros sanitários a montante de sistemas biológicos de tratamento é especialmente atrativa, uma vez que o efluente bruto possui, normalmente, valores de $\mathrm{pH}$ elevados ( $\mathrm{pH}>8,5)$, o que torna desnecessária a adição de alcalinizante e, de certo modo, desonera o processo de tratamento. Portanto, ao longo do experimento, o valor do $\mathrm{pH}$ na massa líquida era aquele do próprio lixiviado bruto em ambos os sistemas de tratamento.

Para o controle do processo de tratamento, eram coletadas alíquotas dos conteúdos dos reatores em intervalos de tempo regulares e a operação de stripping era interrompida quando a concentração de

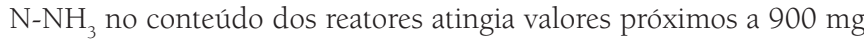
$\mathrm{N}-\mathrm{NH}_{3} \cdot \mathrm{L}^{-1}$. Essa decisão foi norteada pela necessidade de manutenção de condições que favorecessem a remoção biológica do nitrogênio 
através da nitritação/desnitritação no sistema de lodo ativado que recebia o efluente do sistema de stripping. A inibição da conversão bio-

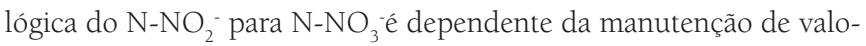
res elevados de $\mathrm{pH}$ no conteúdo do reator. O tratamento do lixiviado bruto resulta em um consumo excessivo da alcalinidade do meio durante a etapa de nitrificação, o que resulta em redução significativa do $\mathrm{pH}$ e, consequentemente, não permite o acúmulo sustentado de

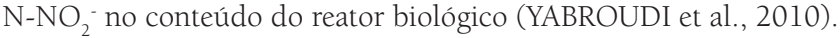

Todas as análises foram realizadas de acordo com APHA, AWWA, WEF (2005). A concentração de carbono orgânico total foi quantificada através da digestão ácida com persulfato de sódio aquecido.

\section{Experimento 2 - Pós-tratamento de lixiviado de aterro através do processo de coagulação- floculação-sedimentação}

O segundo experimento objetivou a avaliação da aplicação do processo de coagulação-floculação-sedimentação para remoção da matéria orgânica recalcitrante presente no efluente de um sistema de lodo ativado operado em bateladas sequenciais alimentado com lixiviado bruto oriundo do aterro São João.

O aterro São João localiza-se no bairro do Sapopemba, na zona leste do município de São Paulo e recebeu cerca de 6.000 t.d de $^{-1}$ de resíduos urbanos, ao longo de 15 anos de operação (entre os anos de 1992 e 2007). Desde a data da ativação até o final da vida útil, recebeu aproximadamente 27,9 milhões de toneladas de resíduos e a quantidade de lixiviado gerado, atualmente, ultrapassa $1.800 \mathrm{~m}^{3} \cdot \mathrm{d}^{-1}$.

O sistema de lodo ativado foi concebido para a remoção da matéria orgânica biodegradável e do nitrogênio amoniacal (nitrificaçãodesnitrificação) presentes no lixiviado bruto. O reator $(42 \times 42 \times 66 \mathrm{~cm})$ foi construído em chapas de acrílico (3,0 mm de espessura) e operou com volume útil igual a 70,0 L. O efluente final do tratamento biológico foi submetido a um tratamento físico-químico (coagulaçãofloculação-sedimentação) prioritariamente concebido para remoção da matéria orgânica recalcitrante.

Os ensaios para avaliação do processo físico-químico de tratamento foram realizados em equipamento de testes de jarros (Jar-test) composto por cinco jarros construídos em acrílico cada um com volume igual a 1,0 L; controlador de rotação; hastes de pás planas construídas em aço inox.

A investigação foi dividida em duas etapas. Durante a primeira, avaliou-se a utilização do cloreto férrico anidro $\left(\mathrm{FeCl}_{3}\right)$ como coagulante, já na segunda etapa, avaliou-se a aplicação do sulfato de alumínio hexahidratado $\mathrm{Al}_{2}\left(\mathrm{SO}_{4}\right) \cdot 6 \mathrm{H}_{2} \mathrm{O}$ como agente coagulante.

\section{$1^{\mathrm{a}}$ Etapa - Testes realizados aplicando $\mathrm{FeCl}_{3}$ como coagulante}

Essa etapa da investigação foi subdividida em três fases. Durante a primeira, investigou-se o uso do $\mathrm{FeCl}_{3}$ variando apenas a dosagem do sal, sem correção do pH do conteúdo dos jarros. Ao longo da segunda fase, variou-se a dosagem do coagulante e o pH das amostras contidas nos jarros. Durante a terceira fase, estudou-se a influência das condições de mistura tais como: gradiente de velocidade e o tempo de mistura sobre as eficiências de remoção de matéria orgânica recalcitrante.

Para a obtenção da dosagem de solução alcalinizante ou ácida necessária para correção do pH de dosagem do conteúdo dos frascos, construiu-se uma curva de correção do pH utilizando um béquer, preenchido com a amostra do efluente do tratamento biológico (1,0 L) à qual acrescentava-se o coagulante com a mesma dosagem que seria utilizada no teste de jarro. Posteriormente, com auxílio de um pHmetro (Orion 720 A), adicionava-se ácido ou álcali até a obtenção do valor de pH desejado, anotando o volume despendido para posterior acréscimo nos jarros. O ajuste do pH era realizado com solução de ácido sulfúrico $\left(\mathrm{H}_{2} \mathrm{SO}_{4}\right)$ 3,0 M ou solução de hidróxido de sódio ( $\mathrm{NaOH}$ ) 6,0M.

O principal parâmetro escolhido para medição da remoção da matéria orgânica recalcitrante foi a cor aparente. Uma porção significativa da matéria orgânica recalcitrante presente em líquidos percolados de aterros sanitários estabilizados é formada por substâncias húmicas, principalmente ácidos húmicos. Essas substâncias são responsáveis pela conferência de cor pronunciada ao despejo (AMOKRAME, COMEL e VERON, 1997; OZTURK et al., 2003; ZOUBOULIS, CHAI e KATSOYIANNIS, 2004; AZIZ et al., 2007). Portanto, o parâmetro cor aparente fornece subsídio para avaliação indireta da presença da matéria orgânica recalcitrante em lixiviados de aterros sanitários.

\section{$2^{\mathrm{a}}$ Etapa - Testes realizados aplicando $\mathrm{Al}_{2}\left(\mathrm{SO}_{4}\right) \cdot 6 \mathrm{H}_{2} \mathrm{O}$ como coagulante}

Ao longo da segunda etapa, os testes seguiram a mesma metodologia utilizada durante aquela conduzida com a aplicação de $\mathrm{FeCl}_{3}$ como coagulante. A cor aparente continuou sendo utilizada como parâmetro para avaliação da remoção da matéria orgânica recalcitrante, entretanto, a aquisição de equipamento específico permitiu a realização de análises de carbono orgânico total (COT) em alíquotas retiradas do conteúdo dos jarros. As análises de COT permitiram, efetivamente, quantificar a matéria orgânica removida através do processo físico-químico de tratamento.

Para manipulação do pH do efluente utilizaram-se as mesmas soluções de alcalinizante e ácido utilizadas para ajuste do pH de dosagem ao longo dos testes de coagulação-floculação-sedimentação.

\section{Resultados e discussão}

\section{Experimento 1 - Pré-tratamento através da operação de stripping da amônia}

$\mathrm{O}$ aterro Bandeirantes é considerado um aterro maduro (aproximadamente 30 anos de operação), consequentemente, o lixiviado 
gerado nesse aterro possui uma concentração elevada de compostos recalcitrantes (relação $\mathrm{DBO} / \mathrm{DQO} \approx 0,3$ ) o que dificulta sua estabilização através de processos biológicos de tratamento. A Tabela 1 mostra as características físico-químicas do lixiviado gerado no aterro Bandeirantes e coletado entre maio/2008 e fevereiro/2009.

Tanto o reator submetido à agitação mecânica, quanto a coluna aerada eram alimentados em batelada, após essa etapa de enchimento eram acionados os dispositivos de agitação (reator em acrílico) e aeração (coluna).

As taxas de remoção de nitrogênio amoniacal variaram entre 3,5 e 3,6 mg N-NH. $\mathrm{L}_{3} \cdot \mathrm{L}^{-1} \cdot \mathrm{h}^{-1}$ para o sistema de stripping composto por um reator submetido à agitação mecânica e entre 3,2 e 3,3 mg N-NH$\cdot \mathrm{LL}^{-1} \cdot \mathrm{h}^{-1}$ para o reator submetido à aeração com bolhas grossas. O desempenho de um sistema de stripping de amônia é influenciada pelo $\mathrm{pH}$, temperatura do meio, vazão de ar aplicada e condições de agitação. Entretanto, o valor do $\mathrm{pH}$, certamente, é um dos fatores mais relevantes para a obtenção de elevadas eficiências de remoção. Marttinen et al. (2002) operaram uma coluna de aeração preenchida com anéis plásticos $\left(Q_{\mathrm{ar}}=10 \mathrm{~L} \cdot \mathrm{h}^{-1}\right)$ e obtiveram taxas de remoção entre 1,0 e 3,0 mg N-NH$H_{3} \cdot \mathrm{L}^{-1} \cdot \mathrm{h}^{-1}$ para valores de $\mathrm{pH}$ entre 7,2 e 9,1. A simples elevação do $\mathrm{pH}$ do meio $(\mathrm{pH}=11,0)$ permitiu aos pesquisadores obterem taxas de remoção entre 6,0 e 9,0 mg N-NH $\mathrm{L}^{-1} \cdot \mathrm{h}^{-1}$, mantendo a temperatura próxima de $20^{\circ} \mathrm{C}$.
A Figura 1 mostra os perfis das concentrações de $\mathrm{N}^{-\mathrm{NH}_{3}}$ ao longo do período de operação dos sistemas de stripping.

Ao longo da condução dos experimentos, em ambos os sistemas, registrou-se uma redução na alcalinidade da água residuária (consumo entre 42 e 58\%). Entretanto, de maneira paradoxal, o valor do $\mathrm{pH}$ do conteúdo dos reatores aumentou de valores próximos a 8,0 para 8,8 , ao longo dos ciclos de tratamento, o que, certamente, representa uma vantagem do processo. Marttinen et al. (2002) também reportaram um aumento do $\mathrm{pH}$ em sistemas de stripping tratando lixiviados de aterros sanitários. Esses pesquisadores registraram que durante as primeiras 6 horas de operação do seu experimento, o valor do $\mathrm{pH}$ rapidamente aumentou de 7,3 para 9,2. Quanto ao acentuado consumo de alcalinidade, trabalhos clássicos atestam que a solubilidade do carbonato de cálcio é significativamente influenciada pela salinidade e pelo potencial iônico do meio (LIJKLEMA, 1972; LINDBERG e BORNO, 1975; SAAS e BEN-YAKOOV, 1977; WERSIN et al., 1989; STUMM e MORGAN, 1996). Lixiviados de aterros sanitários maduros, a exemplo dos utilizados na presente pesquisa, possuem elevada concentração de sais e, provavelmente, tal fato contribuiu para o elevado consumo de alcalinidade ao longo da realização das operações de stripping. a)

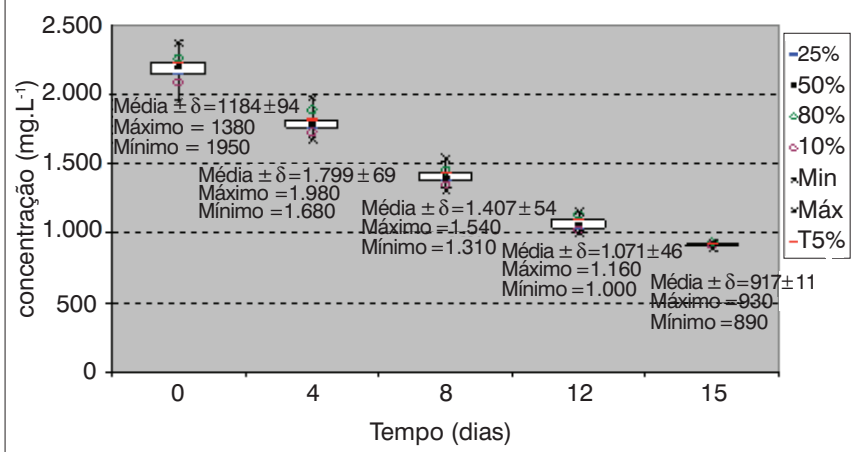

b)

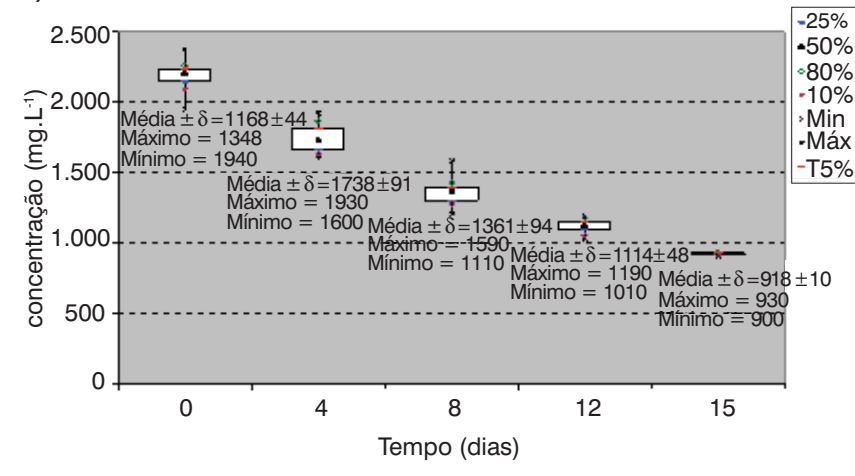

Figura 1 - Perfis temporais das concentrações de $\mathrm{N}_{-} \mathrm{NH}_{3}$ ao longo da operação de stripping conduzida no reator submetido à (a) agitação mecânica $\left(\mathrm{pH}\right.$ inicial $=8,1 ; \mathrm{G}=340 \mathrm{~s}^{-1}$; temperatura entre $22-26^{\circ} \mathrm{C}^{2} \mathrm{n}^{0}$ de ensaios $\left.=20\right)$; (b) aeração com bolhas grossas $\left(Q_{\mathrm{ar}}=30 \mathrm{~L}^{\mathrm{h}} \mathrm{h}^{-1}\right.$; temperatura entre $22-26^{\circ} \mathrm{C} ; \mathrm{pH}$ inicial $=8,1 ; \mathrm{H}_{\text {coluna liquida }}=0,65 \mathrm{~m} ; \mathrm{n}^{0}$ de ensaios $=20$ ).

Tabela 1 - Características físico-químicas do lixiviado gerado no aterro Bandeirantes

\begin{tabular}{|c|c|c|c|c|}
\hline Parâmetro & $\mathrm{n}^{\circ}$ de dados & Valor mínimo & Valor máximo & Valor médio $\pm \delta$ \\
\hline $\mathrm{DQO}\left(\mathrm{mg} \mathrm{O}_{2} \cdot \mathrm{L}^{-1}\right)$ & 20 & 6.800 & 8.000 & $7.373 \pm 372$ \\
\hline $\mathrm{DBO}\left(\mathrm{mg} \mathrm{O}_{2} \cdot \mathrm{L}^{-1}\right)$ & 20 & 1.800 & 2.500 & $2.060 \pm 185$ \\
\hline $\begin{array}{l}\text { Nitrogênio total Kjeldahl } \\
\left(\mathrm{mg} \text { NKT.L }{ }^{-1}\right)\end{array}$ & 20 & 2.050 & 2.580 & $2.343 \pm 114$ \\
\hline $\begin{array}{l}\text { Nitrogênio amoniacal } \\
\left(\mathrm{mg} \mathrm{N}-\mathrm{NH}_{3} \cdot \mathrm{L}^{-1}\right)\end{array}$ & 20 & 1.950 & 2.380 & $2.183 \pm 93$ \\
\hline Carbono orgânico total (mg. $\mathrm{L}^{-1}$ ) & 11 & 1.560 & 2.230 & $1.942 \pm 198$ \\
\hline $\mathrm{pH}$ & 20 & 7,9 & 8,3 & 8,1 \\
\hline Alcalinidade $\left(\mathrm{mg} \mathrm{CaCO}_{3} \cdot \mathrm{L}^{-1}\right)$ & 20 & 9.650 & 12.448 & $10.720 \pm 784$ \\
\hline Sólidos em suspensão totais (mg SST. $\mathrm{L}^{-1}$ ) & 20 & 14.350 & 21.340 & $18.155 \pm 1.676$ \\
\hline Sólidos em suspensão voláteis (mg SSV.L-1) & 20 & 40 & 130 & $92 \pm 22$ \\
\hline
\end{tabular}


Ao longo do processo de tratamento, observou-se uma redução na concentração de matéria orgânica do lixiviado submetido ao stripping. No sistema submetido à agitação mecânica houve uma remoção de COT entre 26 e 31\%, enquanto no reator cilíndrico sujeito à aeração, a remoção esteve compreendida entre 28 e 34\%. Tal fato está associado à presença de compostos orgânicos voláteis, mas observando os resultados de remoção de DBO, DQO e a concentração de SSV apresentados na Tabela 2 , não se deve negligenciar a possibilidade de ocorrência de algum nível de biodegradação devido ao longo tempo de realização da operação de stripping (15 dias). Bloor e Banks (2005) variando a taxa de aplicação de ar entre 1 e 5 litros por minuto em uma coluna aerada preenchida com anéis plásticos e alimentada com lixiviado bruto, obtiveram significativa redução da matéria orgânica presente no despejo (DQO do efluente final $\left.<50 \mathrm{mg} \mathrm{O}_{2} \cdot \mathrm{L}^{-1}\right)$.

\section{Experimento 2- Pós-tratamento de lixiviado de aterro através do processo de coagulação- floculação-sedimentação}

O segundo experimento foi realizado com o lixiviado oriundo do aterro São João e pré-tratado em um sistema de lodo ativado operado em bateladas sequenciais. A Tabela 3 mostra os resultados da caracterização do lixiviado bruto coletado no período compreendido entre os meses março/2008 e janeiro/2009.

O lixiviado bruto era aplicado no sistema de lodo ativado operado em bateladas sequenciais obedecendo a uma razão de troca volumétrica igual a 10\%, ou seja, 7,0 L de lixiviado para 70,0 L de volume útil do reator. A sequência das etapas do tratamento biológico era: alimentação - reação anóxica - aeração - sedimentação - descarte do sobrenadante. Após a etapa de sedimentação, o sobrenadante do reator era coletado e disposto no equipamento de jar-teste para realização do pós-tratamento físico-químico. A Tabela 4 mostra as características do efluente final do tratamento biológico.

\section{$1^{\mathrm{a}}$ Etapa - Testes realizados aplicando $\mathrm{FeCl}_{3}$ como coagulante}

Inicialmente, optou-se por pós-tratar o lixiviado com o $\mathrm{FeCl}_{3}$ sem realizar a correção do pH do meio.Estudos anteriores (MARAÑÓN et al., 2008) atestam a possibilidade de remover a matéria orgânica recalcitrante de lixiviados de aterro sanitário sem a necessidade primordial de correção do pH do meio. Entretanto, naturalmente, a adição de $\mathrm{FeCl}_{3}$ acarreta um consumo de alcalinidade e consequente redução do valor do $\mathrm{pH}$ do conteúdo dos jarros. Quanto às condições de mistura, vale ressaltar que essa etapa foi conduzida considerando fenômenos clássicos de agregação de partículas e, portanto, aplicou-se uma mistura rápida (900 s-1 durante 1 minuto) e uma mistura lenta sem escalonamento de velocidade ( $25 \mathrm{~s}^{-1}$ durante 30 minutos). Observou-se que as melhores remoções ocorreram para dosagens superiores a $300 \mathrm{mg} \mathrm{Fe} \mathrm{F}^{+3} \cdot \mathrm{L}^{-1}$ e valor de $\mathrm{pH}$ do conteúdo dos jarros inferior a 6,0.

A partir dos testes realizados ao longo da primeira fase, verificouse uma influência significativa do valor do pH sobre o fenômeno de coagulação. Portanto, durante a segunda etapa buscou-se obter os

Tabela 2 - Concentrações e eficiências de remoção de matéria orgânica e sólidos em suspensão voláteis nos reatores

\begin{tabular}{|c|c|c|c|c|c|c|c|c|c|}
\hline & \multicolumn{3}{|c|}{$\mathrm{DQO}\left(\mathrm{mg} \mathrm{O}_{2} \cdot \mathrm{L}^{-1}\right)$} & \multicolumn{3}{|c|}{$\mathrm{DBO}\left(\mathrm{mg} \mathrm{O}_{2} \cdot \mathrm{L}^{-1}\right)$} & \multicolumn{3}{|c|}{ (mg SSV.L-1) } \\
\hline & Mín. & Máx. & Média $\pm \delta$ & Mín. & Máx. & Média $\pm \delta$ & Mín. & Máx. & Média $\pm \delta$ \\
\hline Afluente & 6.800 & 8.000 & $7.373 \pm 372$ & 1.800 & 2.500 & $2.060 \pm 185$ & 40 & 130 & $92 \pm 22$ \\
\hline Efluente - R1 & 3.760 & 5.780 & $4.361 \pm 465$ & 790 & 980 & $861 \pm 55$ & 150 & 250 & $202 \pm 32$ \\
\hline Efluente - C1 & 3.200 & 5.510 & $4.382 \pm 500$ & 610 & 900 & $747 \pm 77$ & 180 & 265 & $219 \pm 55$ \\
\hline \multicolumn{10}{|c|}{ Eficiências de remoção (\%) } \\
\hline & \multicolumn{5}{|c|}{ DQO } & \multicolumn{4}{|c|}{ DBO } \\
\hline & \multicolumn{2}{|c|}{ Mín. } & Máx. & \multicolumn{2}{|c|}{ Média $\pm \delta$} & Mín. & \multicolumn{2}{|c|}{ Máx. } & Média $\pm \delta$ \\
\hline R1 & \multicolumn{2}{|c|}{25,9} & 45,4 & \multicolumn{2}{|c|}{$37,2 \pm 5,5$} & 53,4 & \multicolumn{2}{|c|}{65,3} & $58,2 \pm 3,2$ \\
\hline C1 & \multicolumn{2}{|c|}{26,7} & 53,6 & \multicolumn{2}{|c|}{$40,9 \pm 6,3$} & 54,5 & \multicolumn{2}{|c|}{70,0} & $63,7 \pm 4,2$ \\
\hline
\end{tabular}

SSV: sólidos em suspensão voláteis; R1: reator submetido à agitação mecânica; C1: coluna aerada

Tabela 3 - Características físico-químicas do lixiviado gerado no aterro São João

\begin{tabular}{|c|c|c|c|c|}
\hline Parâmetro & $\mathrm{n}^{\circ}$ de dados & Valor mínimo & Valor máximo & Valor médio $\pm \delta$ \\
\hline $\mathrm{DQO}\left(\mathrm{mg} \mathrm{O}_{2} \cdot \mathrm{L}^{-1}\right)$ & 18 & 2.500 & 9.143 & $6.707 \pm 1.605$ \\
\hline $\mathrm{DBO}\left(\mathrm{mg} \mathrm{O}_{2} \cdot \mathrm{L}^{-1}\right)$ & 29 & 515 & 6.138 & $2.409 \pm 1.090$ \\
\hline $\begin{array}{l}\text { Nitrogênio total Kjeldahl } \\
(\mathrm{mg} \text { NKT.L-1) }\end{array}$ & 25 & 1.523 & 2.985 & $2.571 \pm 331$ \\
\hline $\begin{array}{l}\text { Nitrogênio amoniacal } \\
\left(\mathrm{mg} \mathrm{N}-\mathrm{NH}_{3} \cdot \mathrm{L}^{-1}\right)\end{array}$ & 30 & 258 & 2.870 & $2.252 \pm 470$ \\
\hline Carbono orgânico total (mg. $\mathrm{L}^{-1}$ ) & 27 & 943 & 6.610 & $2.384 \pm 1.401$ \\
\hline $\mathrm{pH}$ & 30 & 8,0 & 8,5 & $8,3 \pm 0,1$ \\
\hline Alcalinidade $\left(\mathrm{mg} \mathrm{CaCO}_{3} \cdot \mathrm{L}^{-1}\right)$ & 30 & 6.460 & 14.300 & $10.680 \pm 1.816$ \\
\hline Sólidos em suspensão totais (mg SST.L-1) & 29 & 1.420 & 6.940 & $3.665 \pm 1.204$ \\
\hline Sólidos em suspensão voláteis (mg SSV.L'-1) & 30 & 27 & 370 & $162 \pm 96$ \\
\hline
\end{tabular}


pontos definidos pelos valores de $\mathrm{pH}$ versus dosagens de coagulante, que proporcionassem a melhor remoção de matéria orgânica recalcitrante (avaliada como cor aparente) do efluente do tratamento biológico. Ao longo da etapa anterior, observou-se que os flocos formados apresentavam baixa densidade e não se agrupavam durante a promoção da mistura lenta, portanto, para realização da segunda etapa, ajustou-se o gradiente de mistura para $155 \mathrm{~s}^{-1}$ durante $20 \mathrm{mi}$ nutos. A Figura 2 mostra os resultados obtidos a partir dos testes realizados ao longo da segunda etapa. Os resultados indicam que dosagens acima de $400 \mathrm{mg} \mathrm{Fe} \mathrm{F}^{+3} \cdot \mathrm{L}^{-1}$ não representam um ganho de eficiência justificável na remoção da matéria orgânica recalcitrante. Aziz et al., (2007) também encontraram uma dosagem ótima de cloreto férrico ( $800 \mathrm{mg} \mathrm{FeCl}_{3} . \mathrm{L}^{-1}$ ) acima da qual o processo perde eficiência. Observa-se, ainda, que a melhor eficiência de remoção de cor aparente encontra-se na faixa de $\mathrm{pH}$ compreendida entre 4,0 e 5,0. Aziz et al., (2007) consagraram o pH ótimo de dosagem igual a 4,0. Os pesquisadores afirmaram que em $\mathrm{pH}$ 6,0, foi necessária uma dosagem de $\mathrm{FeCl}_{3}$ igual a $2.500 \mathrm{mg} \cdot \mathrm{L}^{-1}$ para alcançar uma remoção de cor de $97 \%$, enquanto $800 \mathrm{mg} \cdot \mathrm{L}^{-1} \mathrm{de} \mathrm{FeCl}_{3}$ em pH igual a 4,0 permitiu a remoção de 94\% da cor aparente.

Outros trabalhos, também confirmaram que as melhores remoções de matéria orgânica de diferentes percolados de aterros sanitários (medidas como DQO) ocorreram em valores de $\mathrm{pH}$ entre 4,0 e 5,0 (DIAMADOPOULOS, 1994; AMOKRANE, COMEL e VERON, 1997).

Ao longo da terceira fase, buscou-se avaliar a influência das condições de mistura (gradiente de velocidade e tempo de mistura) sobre as eficiências de remoção de matéria orgânica recalcitrante. Variou-se o gradiente de velocidade do equipamento $\left(56,155,286,449,643\right.$ e $\left.869 \mathrm{~s}^{-1}\right)$ e o tempo de mistura $(10,15,30,45,60,90,120 \mathrm{~min})$ mantendo a dosagem do coagulante ( $400 \mathrm{mg} \mathrm{Fe}^{+3} \cdot \mathrm{L}^{-1}$ ) e o valor do $\mathrm{pH}$ de dosagem (entre 4,0 e 5,0) aplicados ao longo da segunda fase. A variação das condições de mistura (Figura 3) não apresentou efeito significativo sobre as eficiências de remoção de matéria orgânica recalcitrante. Obedecendo as mesmas condições da segunda fase, a remoção de cor aparente foi sempre superior a 95\% em toda a faixa de variação dos parâmetros.

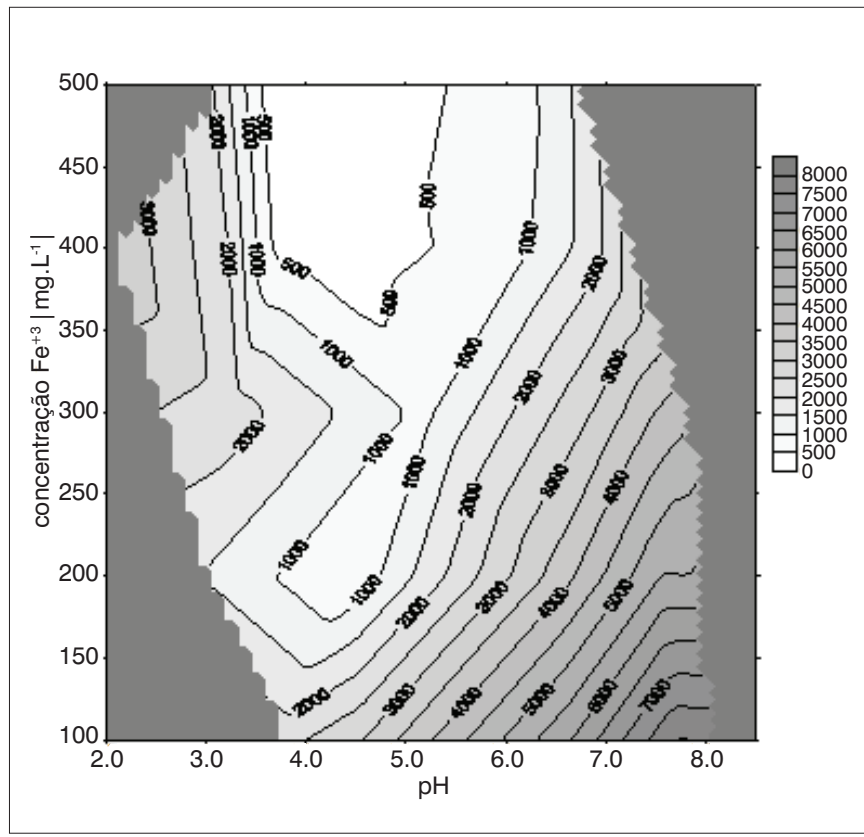

Figura 2 - Cor aparente (unidades Hazen) do efluente final do tratamento físico-químico variando a dosagem do coagulante $\left(\mathrm{FeCl}_{3}\right)$ e 0 valor do $\mathrm{pH}$ do meio líquido.

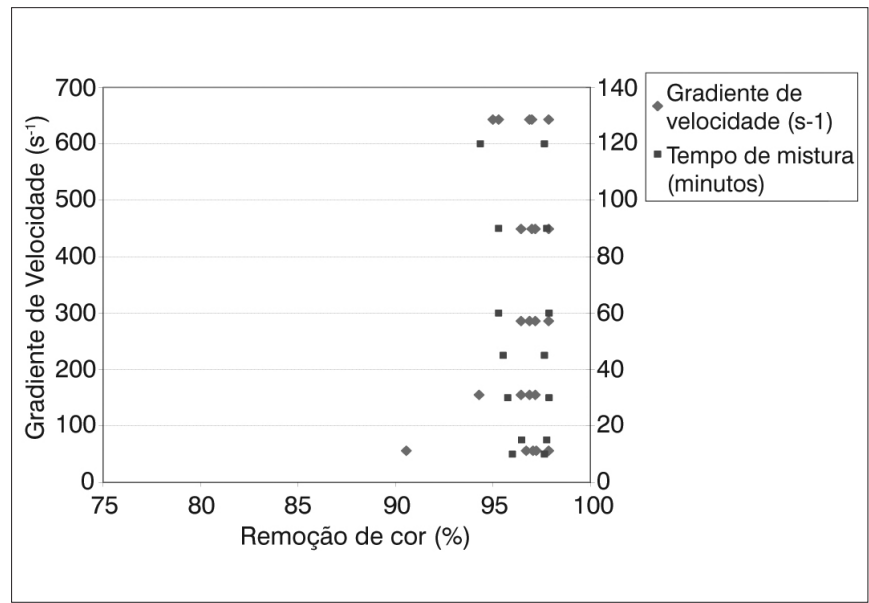

Figura 3 - Eficiências de remoção de matéria orgânica recalcitrante (avaliada como cor aparente) para diferentes valores de gradientes de velocidade e tempo de mistura.

Tabela 4 - Características do efluente do sistema de lodo ativado concebido para o tratamento do lixiviado do aterro São João

\begin{tabular}{|c|c|c|c|c|}
\hline Parâmetro & $\mathrm{n}^{\circ}$ de dados & Valor mínimo & Valor máximo & Valor médio $\pm \delta$ \\
\hline $\mathrm{DQO}\left(\mathrm{mg} \mathrm{O}_{2} \cdot \mathrm{L}^{-1}\right)$ & 21 & 518 & 4.364 & $1.415 \pm 1.086$ \\
\hline $\mathrm{DBO}\left(\mathrm{mg} \mathrm{O}_{2} \cdot \mathrm{L}^{-1}\right)$ & 39 & 3,0 & 126 & $37 \pm 24$ \\
\hline $\begin{array}{l}\text { Nitrogênio amoniacal } \\
\left(\mathrm{mg} \mathrm{N}-\mathrm{NH}_{3} \mathrm{~L}^{-1}\right)\end{array}$ & 35 & n.d. & n.d. & n.d. \\
\hline Carbono orgânico total $\left(\mathrm{mg}^{\mathrm{L}} \mathrm{L}^{-1}\right)$ & 41 & 315 & 2.629 & $838 \pm 410$ \\
\hline $\mathrm{pH}$ & 49 & 6,5 & 8,9 & $8,4 \pm 0,4$ \\
\hline Alcalinidade $\left(\mathrm{mg} \mathrm{CaCO}_{3} \cdot \mathrm{L}^{-1}\right)$ & 43 & 240 & 3.520 & $1.926 \pm 695$ \\
\hline Sólidos em suspensão totais (mg SST.L-1) & 45 & 124 & 1340 & $324 \pm 22$ \\
\hline Sólidos em suspensão voláteis (mg SSV. L $^{-1}$ ) & 45 & 84 & 720 & $184 \pm 117$ \\
\hline Cor aparente $(\mathrm{uH})$ & 43 & 2.500 & 10.000 & $7.500 \pm 1500$ \\
\hline $\mathrm{P}^{-\mathrm{PO}_{4}}{ }^{-3}\left(\mathrm{mg} \cdot \mathrm{L}^{-1}\right)$ & 43 & 1,0 & 12,0 & $4,0 \pm 4,0$ \\
\hline
\end{tabular}

n.d.=valor inferior ao limite de detecção do método analítico empregado; $u H=$ unidades Hazen 


\section{$2^{\mathrm{a}}$ Etapa - Testes realizados aplicando $\mathrm{Al}_{2}\left(\mathrm{SO}_{4}\right)_{3} \cdot 6 \mathrm{H}_{2} \mathrm{O}$ como coagulante}

Como os melhores resultados de remoção de matéria orgânica recalcitrante aplicando $\mathrm{FeCl}_{3}$ como coagulante estiveram associados ao par de valores dosagem versus pH ótimo, a investigação da aplicação do $\mathrm{Al}_{2}\left(\mathrm{SO}_{4}\right)_{3} \cdot 6 \mathrm{H}_{2}$ Ocomo coagulante foi realizada fixando a dosagem e variando o $\mathrm{pH}$ do conteúdo dos jarros em testes sucessivos. As condições de mistura aplicadas foram as mesmas da etapa conduzida com $\mathrm{FeCl}_{3}$ como coagulante.

Os resultados de cor aparente do efluente tratado estão apresentados na Figura 4. Ao longo dessa etapa da investigação, o pH variou entre 3,0 e 8,0 e a dosagem do sulfato de alumínio variou de 100 a $700 \mathrm{mg} \mathrm{Al}{ }^{+3} \cdot \mathrm{L}^{-1}$.

Assim como os resultados obtidos durante a primeira fase, as melhores eficiências de remoção da matéria orgânica recalcitrante foram obtidas para dosagens superiores a $400 \mathrm{mg} \mathrm{Al}^{+3} \cdot \mathrm{L}^{-1}$ e para valores de $\mathrm{pH}$ compreendidos entre 5,0 e 6,0.

Entretanto, expressando os resultados em termos de concentração dos sais aplicados, observa-se que o $\mathrm{FeCl}_{3}$ foi mais eficaz para remoção da matéria orgânica recalcitrante, uma vez que foram dosadas $1.160 \mathrm{mg} \mathrm{FeCl}{ }_{3} \cdot \mathrm{L}^{-1}$, enquanto para obter eficiências de remoção expressivas de matéria orgânica recalcitrante foram necessárias 3.244 mg $\mathrm{Al}_{2}\left(\mathrm{SO}_{4}\right)_{3} \cdot 6 \mathrm{H}_{2} \mathrm{O} \cdot \mathrm{L}^{-1}$.

Esses resultados estão de acordo com outros trabalhos que aplicaram processo de coagulação - floculação (sais de alumínio e ferro) para remoção da matéria orgânica recalcitrante de lixiviados de aterros sanitários. Os pesquisadores afirmaram que o $\mathrm{FeCl}_{3}$ foi mais eficaz para remoção de compostos orgânicos (medida como DQO) de lixiviados de aterros (DIAMADOPOULOS, 1994; AMOKRANE, COMEL e VERON, 1997; TATSI et al., 2003; KARGI e PAMUKOGLU, 2003).

Quanto à produção de lodo do processo, tanto utilizando o $\mathrm{FeCl}_{3}$, quanto o $\mathrm{Al}_{2}\left(\mathrm{SO}_{4}\right)_{3} \cdot 6 \mathrm{H}_{2} \mathrm{O}$ como coagulante confirma-se assertiva de que maiores dosagens implicam em uma maior produção de lodo. A Figura 5 mostra a produção de lodo (avaliada como sólidos em suspensão totais) a partir da dosagem de cloreto férrico. Há que se considerar os custos envolvidos com a desidratação e disposição adequada desse resíduo.

\section{Conclusões}

Visando a remoção de parte da carga de $\mathrm{N}_{-} \mathrm{NH}_{3}$, tanto um reator submetido à agitação mecânica, quanto um reator submetido à aeração com bolhas grossas mostraram-se adequados como opção de pré-tratamento de um lixiviado de aterro sanitário. As taxas de remoção variaram entre 3,5 e 3,6 mg N-NH$\cdot \mathrm{LH}^{-1} \cdot \mathrm{h}^{-1}$ e entre 3,2 e 3,3 mg N-NH${ }_{3} \cdot \mathrm{L}^{-1} \cdot \mathrm{h}^{-1}$, respectivamente.

Ao longo do processo de stripping da amônia, observou-se uma redução na concentração de matéria orgânica presente no lixiviado.
No sistema submetido à agitação mecânica houve uma remoção de COT entre 26 e 31\%, enquanto no reator cilíndrico sujeito à aeração, a remoção esteve compreendida entre 28 e 34\%.

$\mathrm{O}$ tratamento físico-químico pelo processo de coagulação-floculação, utilizando sais de ferro e alumínio apresentou resultados satisfatórios para remoção dos compostos recalcitrantes (avaliada como cor aparente) presentes no efluente de um sistema de lodo ativado tratando lixiviado de aterro sanitário. Foram necessárias dosagens de $400 \mathrm{mg} \mathrm{Fe}^{+3} \cdot \mathrm{L}^{-1} \mathrm{e} 400 \mathrm{mg}$ $\mathrm{Al}^{+3} \cdot \mathrm{L}^{-1}$ para o alcance de remoções superiores a $90 \%$, além do ajuste de valores de $\mathrm{pH}$ de dosagem para valores próximos de 4,0 utilizando como coagulante cloreto férrico, e, no uso do sulfato de alumínio, $\mathrm{pH}$ próximo de 5,0. As condições de mistura como gradiente de velocidade e tempo de mistura, utilizando o par dosagem ótima e pH ideal, não apresentaram influência sobre a remoção da matéria orgânica recalcitrante.

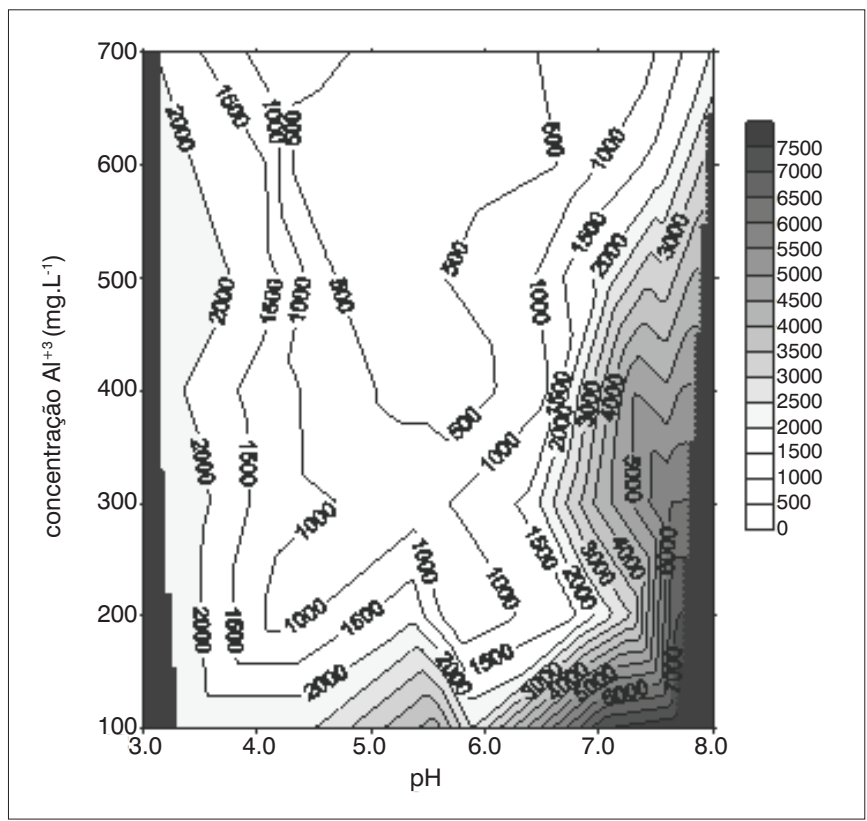

Figura 4 - Cor aparente (unidades Hazen) do efluente final do tratamento físico-químico variando a dosagem do coagulante - $\mathrm{Al}_{2}\left(\mathrm{SO}_{4}\right)_{3} \cdot 6 \mathrm{H}_{2} \mathrm{O}$ - e 0 valor do $\mathrm{pH}$ do meio líquido.

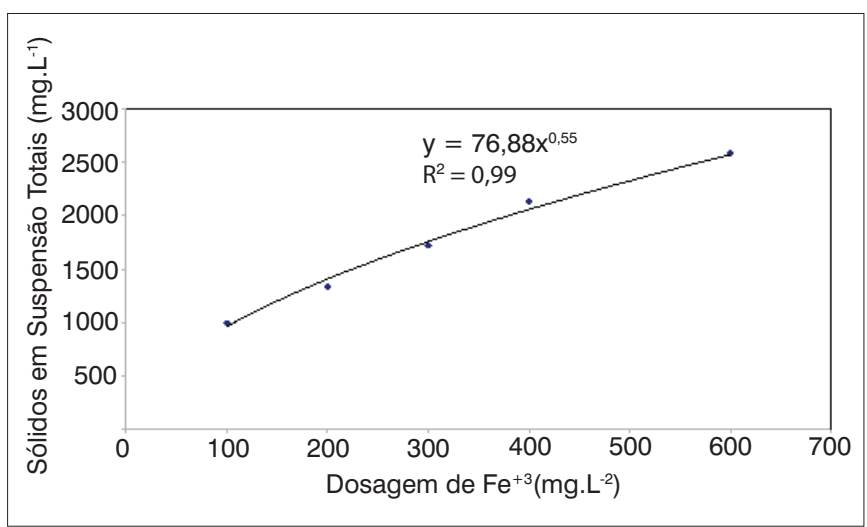

Figura 5 - Curva de produção de lodo químico utilizando coagulante cloreto férrico. 


\section{Referências}

ALEM SOBRINHO, P.; POVINELLI, J. (2009). In: GOMES, L. P. (Coordenadora) Estudos de caracterização e tratabilidade de lixiviados de aterros sanitários para as condições brasileiras. ABES. Rio de Janeiro, p. 19-25.

AMARAL, M.C.S.; FERREIRA, C.F.A.; LANGE, L.C.; et al. (2008) Avaliação da biodegradabilidade anaeróbia de lixiviados de aterro sanitários. Engenharia Sanitária e Ambiental. [online]. v.13, n.1, p. 38-45.

AMERICAN PUBLIC HEALTH ASSOCIATION (APHA); AMERICAN WATER WORKS ASSOCIATION (AWWA); WATER ENVIRONMENTAL FEDERATION (WEF). (2005) Standard methods for the examination of water and wastewater. $21^{\text {st }}$.ed., Washington, EUA

AMOKRANE, A.; COMEL, C; VERON, J. (1997) Landfill leachates pretreatment by coagulation-flocculation. Water Research, v.31, p. 2775-2782.

AZIZ, H.A.; ALIAS, S.; ADLAN, M.N.; et al. (2007) Colour removal from landfill leachate by coagulation and flocculation processes. Bioresource Technology, v. 98, p. 218-220.

BRASIL. Instituto Brasileiro de Geografia e Estatística. (2010) Pesquisa nacional de saneamento básico 2008. Rio de Janeiro. 219 p.

BLOOR, M.C.; BANKS, C.J. (2005) Acute and sub-lethal toxicity of landfill leachate towards two aquatic macro-invertebrates: Demonstrating the remediation potential of air stripping. Environment International, v. 31, p. 1114-1122.

DIAMADOPOULOS, E. (1994) Characterization and treatment of recirculation stabilized leachate. Water Research, v. 28, p. 2439-2445

Instituto de pesquisas tecnológicas/CEMPRE. (2000). Lixo municipal: manual de gerenciamento integrado. 2. ed. São Paulo. 370 p.

KARGI, F.; PAMUKOGLU, M.Y. (2003) Powdered activated carbon added biological treatment of pre-treated landfill leachate in a fed-batch reactor. Biotechnology Letters, v. 25, p. 695-699.

KURNIAWAN, T.A.; LO, W.;CHAN, G.Y.S. (2006) Physico-chemical treatments for removal of recalcitrant contaminants from landfill leachate. Journal of Hazardous Materials, B129, p. 80-100.
LIJKLEMA, L. (1972) Factors affecting pH change in alkaline waste water treatment - III: A dynamic simulation. Water Research, v. 6 p. $165-182$

Lindberg, A.; Borno, C. (1975) Calculation of Calcium Carbonate-Carbon Dioxide Equilibrium in Water and Mixtures of Water. Verhandlungen Internationale Vereinigung Limnologie, v. 19, p. 1511-1517.

Marañón, E.; Castrillón, L.; Fernández-Nava, Y.; et al. (2008) Coagulationflocculation as a pretreatment process at a landfill leachate nitrificationdenitrification plant. Journal of Hazardous Materials, v. 156, p. 538-544.

Marttinen, S.K.; Kettunenb, R.H.; Sormunena, K.M.; et al. (2002) Screening of physical-chemical methods for removal of organic material, nitrogen and toxicity from low strength landfill leachates. Chemosphere, v. 46, p. $851-858$.

OZTURK, I; ALTINBAS, M.; KOYUNCU, I.; et al. (2003) Advanced physicochemical treatment experiences on young municipal landfill leachates. Waste Management, v. 23, p. 441-446.

SAAS, E.; BEN-YAAKOV, S. (1977) The carbonate system in hypersaline solutions: dead sea brines. Marine Chemistry, v. 5, p. 183-199.

Stumm, W.; Morgan, J.J. (1996) Aquatic Chemistry, Chemical Equilibria and Rates in Natural Waters. $3^{\text {rd }}$ ed. John Wiley \& Sons, Inc., New York, $1022 \mathrm{p}$

Tatsi, A.A.; Zouboulis, A.I.; Matis, K.A., et al. (2003) Coagulationflocculation pretreatment of sanitary landfill leachate. Chemosphere, v. 53, p. $734-744$

Wersin, P.; Charlet, L.; Karthein, R.; et al. (1989) From adsorption to precipitation: Sorption of $\mathrm{Mn}^{2+}$ on $\mathrm{FeCO}_{3(\mathrm{~s})}$. Geochimica et Cosmochimica Acta, v. 53, p. 2787-2796

YABROUDI, S.C.; ALEM SOBRINHO, P.; MORITA, D.M.; et al. (2010) Aplicabilidad del proceso de nitritación/desnitritación en el tratamiento de lixiviado de relleno sanitário. Interciencia (Caracas), v. 35, p. 921-926.

ZOUBOULIS, A.I.; CHAI, X.; KATSOYIANNIS, I.A. (2004) The application of biofloculant for the removal of humic acids from stabilized landfil leachates. Journal of Environmental Management, v. 70, p. 35-41. 